\title{
Machine Learning Techniques for Assistive Robotics
}

\author{
Ester Martinez-Martin ${ }^{+} \mathbb{D}$, Miguel Cazorla ${ }^{*}+\underset{\mathbb{C}}{ }$ and Sergio Orts-Escolano ${ }^{\dagger}$ \\ Institute for Computing Research, University of Alicante, 03690 Alicante, Spain; ester@ua.es (E.M.-M.); \\ sorts@ua.es (S.O.-E.) \\ * Correspondence: miguel.cazorla@ua.es; Tel.: +34-965903400 \\ † These authors contributed equally to this work.
}

Received: 4 May 2020; Accepted: 14 May 2020; Published: 16 May 2020

\section{Introduction}

Assistive robots are a category of robots that share their area of work and interact with humans. Their main goal is to help, assist, and monitor humans, especially people with disabilities. To achieve this goal, it is necessary that these robots possess a series of characteristics: the ability to perceive their environment from their sensors and act consequently, to interact with people in a multimodal manner, and to navigate and make decisions autonomously. This complexity demands computationally expensive algorithms to be performed in real-time. Therefore, with the advent of high-end embedded processors, several algorithms could be processed concurrently and in real-time.

All these capabilities involve, to a greater or less extent, the use of machine learning techniques. In particular, in the last few years, new deep learning techniques have enabled a very important qualitative leap in different problems related to perception, navigation, and human understanding. In this Special Issue, various works are presented involving the use of machine learning techniques for assistive technologies, but in particular for assistive robots.

\section{Machine Learning Techniques for Assistive Robotics}

This Special Issue consists of eleven papers covering the application of machine learning techniques on assistive technologies and assistive robots. There are two review papers and nine research ones.

The first review [1] is focused on the identification of the research works written in English about the recognition of daily activities and environment recognition using the AdaBoost method. In particular, it focuses on the data obtained from the sensors available in mobile devices that were published between 2012 and 2018. The second one [2] reviews and summarizes the research efforts toward the development of these kinds of systems, focusing on two social groups: older adults and children with autism.

Regarding research papers, there are nine, and they are described briefly in the next paragraphs.

Pires et al. [3] use artificial neural networks (ANN) for the recognition of activities of daily living (ADLs) with the data acquired from the sensors available in mobile devices. Firstly, before ANN training, the mobile device is used for data collection. After training, mobile devices are used to apply an ANN previously trained for the ADLs' identification on a less restrictive computational platform.

In Reference [4], a system to detect the performance and the emotional state that elderly people have when performing exercises is presented. With this detection, the authors want to build an assistant that motivates those people to perform exercises and, concurrently, monitors them, observing their physical and emotional responses.

The paper presented by Ferreira et al. [5] proposes the recognition of eight ADL, e.g., walking, running, standing, going upstairs, going downstairs, driving, sleeping, and watching television, and nine environments, e.g., bar, hall, kitchen, library, street, bedroom, living room, gym, and classroom, using the instance-based k-nearest neighbor (IBk) and AdaBoost methods. The primary purpose of this paper is to find the best machine learning method for ADL and environment recognition. 
The main proposal in [6] is to recognize users' environment and standing activities. Furthermore, these features are included in a framework for the ADL and environment identification. Therefore, this paper is divided into two parts: firstly, acoustic sensors are used for the collection of data towards the recognition of the environment, and secondly, the information of the recognized environment is fused with the information gathered by motion and magnetic sensors. The environment and ADL recognition are performed by pattern recognition techniques that aim for the development of their system, including data collection, processing, fusion, and classification procedures.

Modern achievements accomplished in both cognitive neuroscience and human-machine interaction technologies have enhanced the ability to control devices with the human brain by using brain-computer interface systems. In particular, the development of brain-controlled mobile robots is very important because systems of this kind can assist people, suffering from devastating neuromuscular disorders, move and thus improve their quality of life. The research work presented in [7] concerns the development of a system that performs motion control in a mobile robot in accordance with the eye blinking of a human operator via a synchronous and endogenous electroencephalography-based brain-computer interface, which uses alpha brain waveforms. The received signals are filtered in order to extract suitable features. These features are fed as inputs to a neural network, which is properly trained in order to guide the robotic vehicle.

One of the main problems in the elderly population and for people with functional disabilities is falling when they are not being supervised. Therefore, there is a need for monitoring systems with fall detection functionality. Mobile robots are a good solution for keeping the person in sight when compared to static-view sensors. Along this line, Maldonado et al. [8] propose a vision-based solution for fall detection based on a mobile-patrol robot that can correct its position in case of doubt. Deep learning-based computer vision is used for person detection, and fall classification is done by using a learning-based support vector machine (SVM) classifier.

In Reference [9], a Siamese network with an auto-encoding constraint is proposed to extract discriminative features from detection responses in a tracking-by-detection framework. The proposed network is improved to extract the previous-appearance-next vector from the tracklet for better association. Feature experiments show that the proposed Siamese network has advantages in terms of both discrimination and correctness.

Classification of complex acoustic scenes under real-time scenarios is an active domain, which has been engaged by several researchers lately from the machine learning community. In Reference [10], a framework for automatic acoustic classification for behavioral robotics is presented. Motivated by several texture classification algorithms used in computer vision, a modified feature descriptor for sound is proposed, which incorporates a combination of 1D local ternary patterns (1D-LTP) and baseline method Mel-frequency cepstral coefficients (MFCC). The extracted feature vector is later classified using a multi-class SVM, which is selected as a base classifier.

Near-infrared (NIR) facial expression recognition is resistant to illumination change. Chen et al. [11] propose a three-stream three-dimensional convolutional neural network with a squeeze-and-excitation (SE) block for NIR facial expression recognition. Each stream is fed with different local regions, namely the eyes, nose, and mouth. The experimental results on the Oulu-CASIANIR facial expression database show that the proposed method has a higher recognition rate than some of the state-of-the-art algorithms.

\section{Future}

The elderly population is growing year-by-year, and therefore, assistive robotics could help to improve their standard of living and quality of life. Additionally, it can also go beyond this group of people and help others that also currently live with disabilities and impairments. Machine learning techniques will help in developing robust methods in this area, creating products (robots, devices, etc.) and solutions that live together with humans on a daily basis. 
Funding: This research received no external funding.

Conflicts of Interest: The authors declare no conflicts of interest.

\section{Abbreviations}

The following abbreviations are used in this manuscript:

$\begin{array}{ll}\text { ANN } & \text { Artificial neural networks } \\ \text { ADL } & \text { Activities of daily living } \\ \text { IBk } & \text { Instance-based 32k-nearest neighbor } \\ \text { SVM } & \text { Support vector machine } \\ \text { 1D-LTP } & \text { 1D local ternary patterns } \\ \text { MFCC } & \text { Mel-frequency cepstral coefficients } \\ \text { NIR } & \text { Near-infrared } \\ \text { SE } & \text { Squeeze-and-excitation }\end{array}$

\section{References}

1. Ferreira, J.M.; Pires, I.M.; Marques, G.; Garcia, N.M.; Zdravevski, E.; Lameski, P.; Flórez-Revuelta, F.; Spinsante, S. Identification of Daily Activites and Environments Based on the AdaBoost Method Using Mobile Device Data: A Systematic Review. Electronics 2020, 9, 192. [CrossRef]

2. Martinez-Martin, E.; Escalona, F.; Cazorla, M. Socially Assistive Robots for Older Adults and People with Autism: An Overview. Electronics 2020, 9, 367. [CrossRef]

3. Pires, I.M.; Marques, G.; Garcia, N.M.; Pombo, N.; Flórez-Revuelta, F.; Spinsante, S.; Teixeira, M.C.; Zdravevski, E. Recognition of Activities of Daily Living and Environments Using Acoustic Sensors Embedded on Mobile Devices. Electronics 2019, 8, 1499. [CrossRef]

4. Costa, A.; Rincon, J.A.; Julian, V.; Novais, P.; Carrascosa, C. A Low-Cost Cognitive Assistant. Electronics 2020, 9, 310. [CrossRef]

5. Ferreira, J.M.; Pires, I.M.; Marques, G.; García, N.M.; Zdravevski, E.; Lameski, P.; Flórez-Revuelta, F.; Spinsante, S.; $\mathrm{Xu}, \mathrm{L}$. Activities of Daily Living and Environment Recognition Using Mobile Devices: A Comparative Study. Electronics 2020, 9, 180. [CrossRef]

6. Pires, I.M.; Marques, G.; Garcia, N.M.; Flórez-Revuelta, F.; Canavarro Teixeira, M.; Zdravevski, E.; Spinsante, S.; Coimbra, M. Pattern Recognition Techniques for the Identification of Activities of Daily Living Using a Mobile Device Accelerometer. Electronics 2020, 9, 509. [CrossRef]

7. Korovesis.; Kandris.; Koulouras.; Alexandridis. Robot Motion Control via an EEG-Based Brain-Computer Interface by Using Neural Networks and Alpha Brainwaves. Electronics 2019, 8, 1387. [CrossRef]

8. Maldonado-Bascón, S.; Iglesias-Iglesias, C.; Martín-Martín, P.; Lafuente-Arroyo, S. Fallen People Detection Capabilities Using Assistive Robot. Electronics 2019, 8, 915. [CrossRef]

9. Liu, P.; Li, X.; Liu, H.; Fu, Z. Online Learned Siamese Network with Auto-Encoding Constraints for Robust Multi-Object Tracking. Electronics 2019, 8, 595. [CrossRef]

10. Aziz, S.; Awais, M.; Akram, T.; Khan, U.; Alhussein, M.; Aurangzeb, K. Automatic Scene Recognition through Acoustic Classification for Behavioral Robotics. Electronics 2019, 8, 483. [CrossRef]

11. Chen, Y.; Zhang, Z.; Zhong, L.; Chen, T.; Chen, J.; Yu, Y. Three-Stream Convolutional Neural Network with Squeeze-and-Excitation Block for Near-Infrared Facial Expression Recognition. Electronics 2019, 8, 385. [CrossRef]

(C) 2020 by the authors. Licensee MDPI, Basel, Switzerland. This article is an open access article distributed under the terms and conditions of the Creative Commons Attribution (CC BY) license (http:/ / creativecommons.org/licenses/by/4.0/). 